\title{
Based on Network Pharmacology Tools to Investigate the Molecular Mechanism of Cordyceps sinensis on the Treatment of Diabetic Nephropathy
}

\author{
Yan Li, ${ }^{1}$ Lei Wang, ${ }^{2}$ Bojun Xu, ${ }^{1}$ Liangbin $\mathrm{Zhao},{ }^{1} \mathrm{Li} \mathrm{Li},{ }^{1}$ Keyang $\mathrm{Xu},{ }^{3}$ Anqi Tang, \\ Shasha Zhou, ${ }^{1}$ Lu Song, ${ }^{1}$ Xiao Zhang, ${ }^{1}$ and Huakui Zhan ${ }^{1}{ }^{1}$ \\ ${ }^{1}$ Hospital of Chengdu University of Traditional Chinese Medicine, Chengdu, 610072 Sichuan, China \\ ${ }^{2}$ Key Laboratory of Chinese Internal Medicine of Ministry of Education and Dongzhimen Hospital, Beijing University of \\ Chinese Medicine, Beijing 100700, China \\ ${ }^{3}$ Zhejiang Chinese Medical University, Hangzhou, 310053 Zhejiang, China
}

Correspondence should be addressed to Huakui Zhan; zhanhuak@qq.com

Received 27 August 2020; Revised 17 January 2021; Accepted 24 January 2021; Published 8 February 2021

Academic Editor: Michaelangela Barbieri

Copyright (c) 2021 Yan Li et al. This is an open access article distributed under the Creative Commons Attribution License, which permits unrestricted use, distribution, and reproduction in any medium, provided the original work is properly cited.

\begin{abstract}
Background. Diabetic nephropathy (DN) is one of the most common complications of diabetes mellitus and is a major cause of endstage kidney disease. Cordyceps sinensis (Cordyceps, Dong Chong Xia Cao) is a widely applied ingredient for treating patients with DN in China, while the molecular mechanisms remain unclear. This study is aimed at revealing the therapeutic mechanisms of Cordyceps in DN by undertaking a network pharmacology analysis. Materials and Methods. In this study, active ingredients and associated target proteins of Cordyceps sinensis were obtained via Traditional Chinese Medicine Systems Pharmacology Database (TCMSP) and Swiss Target Prediction platform, then reconfirmed by using PubChem databases. The collection of DN-related target genes was based on DisGeNET and GeneCards databases. A DN-Cordyceps common target interaction network was carried out via the STRING database, and the results were integrated and visualized by utilizing Cytoscape software. Gene Ontology (GO) and Kyoto Encyclopedia of Genes and Genomes (KEGG) pathway enrichment analyses were performed to determine the molecular mechanisms and therapeutic effects of Cordyceps on the treatment of DN. Results. Seven active ingredients were screened from Cordyceps, 293 putative target genes were identified, and 85 overlapping targets matched with DN were considered potential therapeutic targets, such as TNF, MAPK1, EGFR, ACE, and CASP3. The results of GO and KEGG analyses revealed that hub targets mainly participated in the AGE-RAGE signaling pathway in diabetic complications, TNF signaling pathway, PI3K-Akt signaling pathway, and IL-17 signaling pathway. These targets were correlated with inflammatory response, apoptosis, oxidative stress, insulin resistance, and other biological processes. Conclusions. Our study showed that Cordyceps is characterized as multicomponent, multitarget, and multichannel. Cordyceps may play a crucial role in the treatment of DN by targeting TNF, MAPK1, EGFR, ACE, and CASP3 signaling and involved in the inflammatory response, apoptosis, oxidative stress, and insulin resistance.
\end{abstract}

\section{Introduction}

Diabetic nephropathy (DN) is featured as hyperglycemia, hyperfiltration, proteinuria, and progressive renal function decline, which can cause end-stage kidney disease [1]. It accounts for about $40 \%$ of chronic kidney disease worldwide and is undoubtedly a medical challenge worldwide [2], in terms of high incidence, multifactorial pathogenesis, and the absence of practical methods in the diagnosis and treatment [3]. Traditional Chinese medicine (TCM) guided by the unique theory provides an effective treatment of complex chronic diseases via multicomponent, multitarget, and multipathway [4]. In recent years, Chinese herb medicine has been commonly utilized to alleviate and reverse diabetes and its complications, such as DN in clinical practice and scientific researches, which has been considered a beneficial supplement 
of the drug therapy for DN [4]. Cordyceps, a traditional Chinese herbal medicine, is reported to have multiple healthpromoting characteristics, including anti-inflammatory, anticancer, antidiabetic, analgesic, antioxidant, antiallergic, and antiobesity [5]. Also, Cordyceps has been reported to have broad pharmacological effects on $\mathrm{DN}$ by inhibiting the epithelial-mesenchymal transition, alleviating oxidative stress, repressing inflammation, modulating gut microbiota dysbiosis, and activating autophagy [6-9]. However, the pharmacological mechanisms of Cordyceps associated with $\mathrm{DN}$ only focus on a single chemical molecule. Accordingly, a comprehensive and systematic evaluation of the molecular mechanisms of Cordyceps on DN is indispensable.

This study is aimed at analyzing the pharmacological mechanisms of active ingredients of Cordyceps involved in the progression of $\mathrm{DN}$ via using the network pharmacology databases and biological analysis methods. It laid a stable foundation for further research on exploring pharmacological mechanisms of Cordyceps in treating DN. The study framework is showed in Figure 1.

\section{Materials and Methods}

2.1. Establishment of Active Ingredients and Correlated Target Database. TCMSP (http://lsp.nwu.edu.cn/tcmsp.php), a systematic pharmacological platform that contains the relationships among herbal compounds, related targets, and diseases [10], was applied to identify the chemical constituents of Cordyceps. The active components were selected based on oral bioavailability $(\mathrm{OB})$ and drug likeness $(\mathrm{DL})$ values, and the ingredients were captured when the OB was $\geq 30 \%$ and the DL $\geq 0.18$ (a screening threshold of TCMSP database) [10]. The chemical formulas of components were reconfirmed by PubChem (https://www.ncbi.nlm.nih.gov/ pccompound) to double-check the final compounds of Cordyceps [11]. The targets associated with Cordyceps components were further identified based on the TCMSP database and Swiss Target Prediction (http://www .swisstargetprediction.ch.), a webserver to accurately identify the targets of active molecules [12]. The genes corresponding to the protein targets were obtained from the UniProt database.

\subsection{Network Construction of Active Components-Potential} Targets. A comprehensive network was constructed via using Cytoscape software to reflect the intricate relationship between active compounds and putative targets [13]. Nodes represent the components and targets, while edges reveal the interactions between components and targets (Figure 2).

2.3. Selection of Potential DN Targets. The keyword "diabetic nephropathy" was inputted in the GeneCards (https://www .genecards.org/), a human gene compendium with information about genomics, proteomics, and transcriptomics [14], and DisGeNET (https://www.disgenet.org/home/), a comprehensive platform including one of the largest publicly accessible collections of genes, to search for DN-associated targets [15].
2.4. Screening Compound-Disease Overlapping Targets. The screened compound targets and disease targets were imported into Funrich, a software used mainly for functional enrichment and interaction network analysis of genes and proteins for analysis [16]. The common targets of compound-disease were obtained as the potential targets for further analysis (Figure 3).

2.5. Network Construction of Compound-Disease Common Targets. A protein-protein interaction (PPI) network was obtained based on the STRING platform (https://string-db .org/), which covers nearly all functional interactions among the expressed proteins [17]. Target interaction information derived from the STRING database was imported into the Cytoscape (version 3.7.1; https://www.cytoscape.org/) software where the interaction information was integrated and analyzed.

2.6. GO and KEGG Pathway Enrichment Analyses. GO is the most comprehensive and widely used knowledgebase for the classification of gene functions, including the biological process (BP), molecular function (MF), and cell component (CC) [18]. KEGG (http://www.kegg.jp/) is an encyclopedia of genes and genomes; connecting genomic information to higher-order functional information to capture significantly enriched biological pathways is the major objective of the KEGG knowledgebase [19].

In our study, GO functional annotation and KEGG pathway enrichment analysis were carried out via using the ClusterProfiler package of $\mathrm{R}$ software, and $P<0.05$ was employed as a screening threshold.

\section{Results}

3.1. Active Ingredients of Cordyceps. The active chemical components of Cordyceps were selected via the TCMSP databases, and 38 compounds were collected with the thresholds of $\mathrm{OB} \geq 30 \%$ and $\mathrm{DL} \geq 0.18$ properties. Finally, 7 candidate ingredients were screened out for further study (Table 1).

3.2. Compound-Target Network Construction. In order to visualize the interaction relationship between the Cordyceps ingredients and corresponding targets, we established a compound-target network (Figure 2). By mapping 7 components to 293 potential targets, the network comprises 300 nodes and 500 edges, in which the red circles correspond to the putative targets and Cordyceps ingredients are in green. Chemical compound arachidonic acid, cerevisterol, betasitosterol, linoleyl acetate, cholesteryl palmitate, CLR, and peroxyergosterol correspond to $133,100,77,57,53,49$, and 31 targets, respectively. The results suggest that these $7 \mathrm{com}$ ponents probably serve significant therapeutic roles in $\mathrm{DN}$.

3.3. Predicting DN-Related Targets. By retrieving the DisGeNET and GeneCards databases, results were integrated to obtain the DN-associated targets. As shown in Figure 3, the potential target genes in Cordyceps were matched to the $\mathrm{DN}$-associated target gene by using the Funrich platform and was shown as a Venn diagram. Finally, 85 putative targets were selected according to the intersection of component 


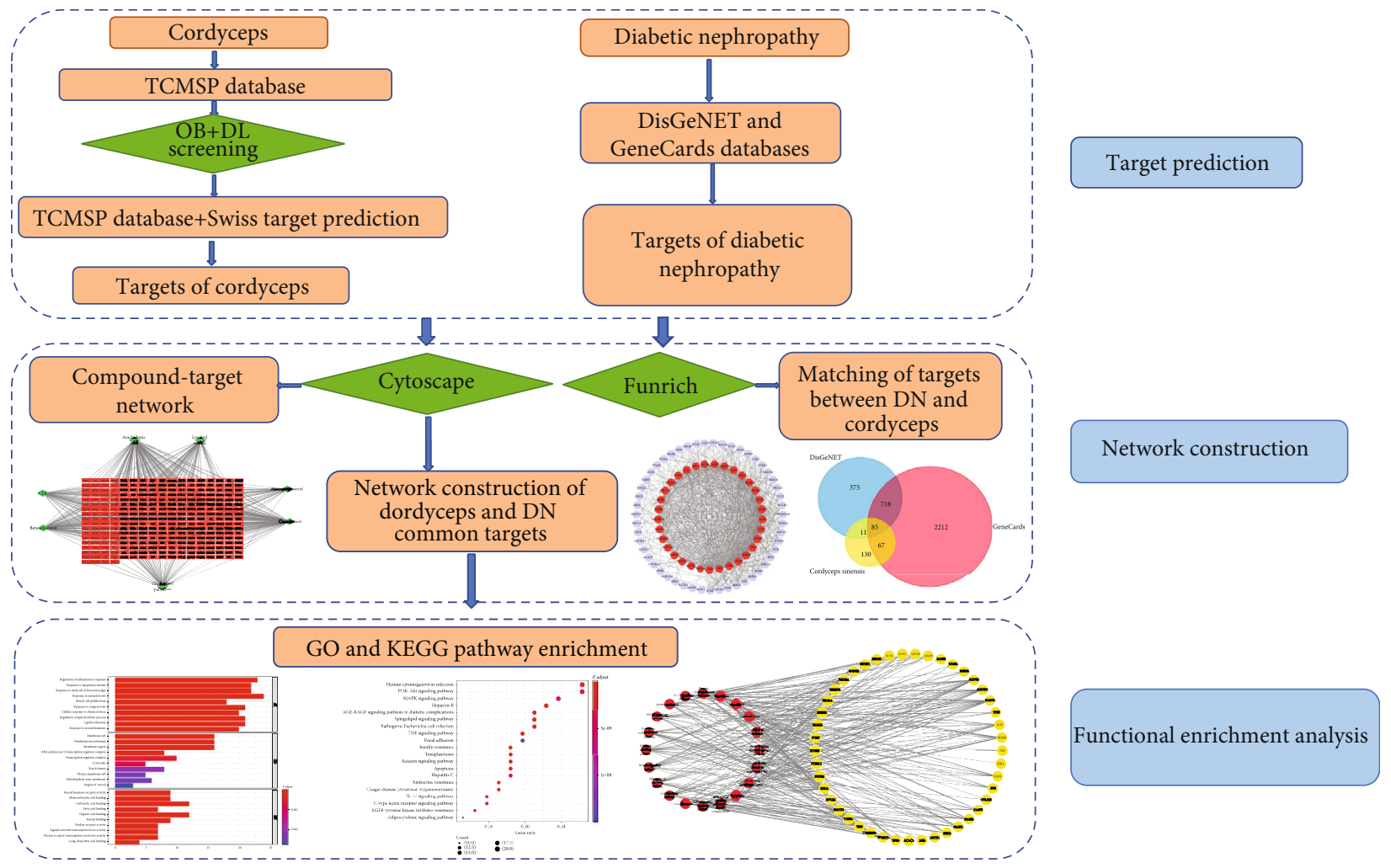

FIGURE 1: A flow chart based on an integration strategy of network pharmacology.

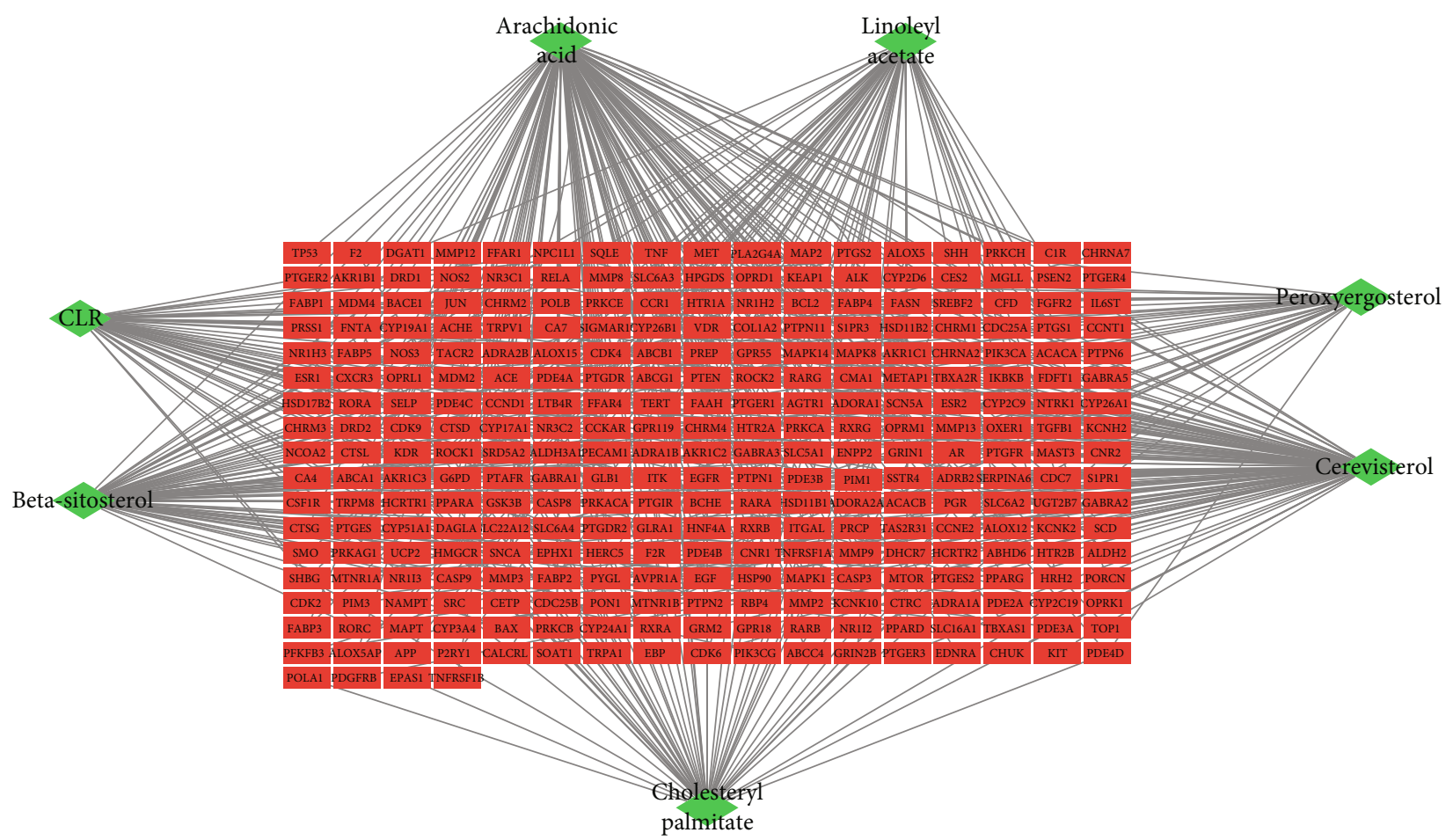

FIGURE 2: Compound-target network of putative targets and active Cordyceps ingredients. The green nodes represent the active components of Cordyceps, and nodes in red represent the corresponding targets of the ingredients. 


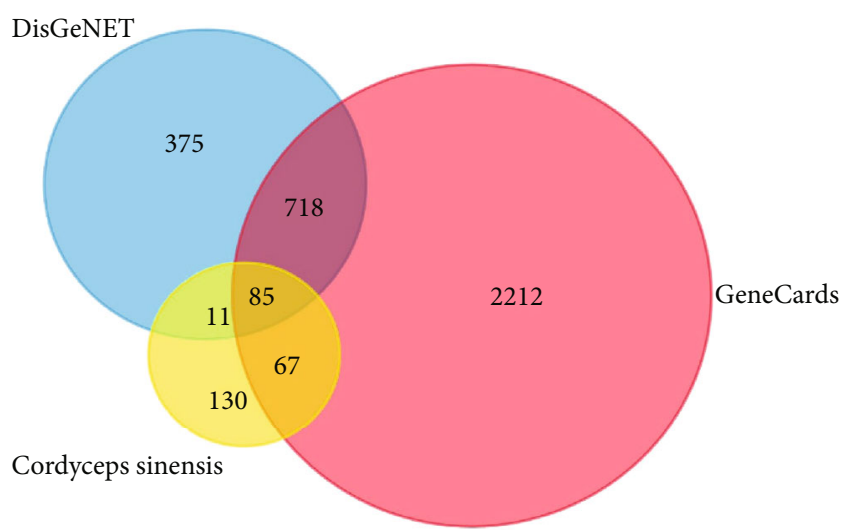

FIgURE 3: Overlapping target genes between DN and Cordyceps.

TABlE 1: Essential information about Cordyceps active components.

\begin{tabular}{lccc}
\hline Mol ID & Molecule name & OB (\%) & DL \\
\hline MOL001645 & Linoleyl acetate & 42.1 & 0.2 \\
MOL001439 & Arachidonic acid & 45.57 & 0.2 \\
MOL008999 & Cholesteryl palmitate & 31.05 & 0.45 \\
MOL000953 & CLR & 37.87 & 0.68 \\
MOL000358 & Beta-sitosterol & 36.91 & 0.75 \\
MOL008998 & Cerevisterol & 39.52 & 0.77 \\
MOL011169 & Peroxyergosterol & 44.39 & 0.82 \\
\hline
\end{tabular}

targets and DN-related targets; one target was excluded because it had no interaction with other targets (Table 2).

3.4. Common Target Network. 85 putative genes correlated with DN were imported to the STRING database for analysis and network establishment. The interaction network was based on the selected targets with a medium confidence score of 0.400 (Figure 4). A total of 84 nodes and 767 edges were embodied, and the average node degree is 18.3 after analysis. The results were imported to the Cytoscape software for further analysis and network construction (Figure 5). In this figure, the edges represent the interaction between a pair of potential targets, while the nodes represent the targets, and the degree value indicates the intensity of target interaction.

3.5. GO and KEGG Pathway Enrichment Analyses. After using the ClusterProfiler package for pathway analysis, a total of 1843 biological processes were enriched. The top 10 remarkably enriched BP terms were selected for analysis, including regulation of inflammatory response, response to lipopolysaccharide, response to molecule of bacterial origin, response to nutrient levels, muscle cell proliferation, response to oxygen levels, cellular response to chemical stress, regulation of lipid metabolic process, and lipid localization. Besides, 32 cell components were enriched, and the top 10 entries were screened, consisting of membrane raft, membrane microdomain, membrane region, RNA polymerase II transcription regulator complex, transcription regulator complex, caveola, vesicle lumen, plasma membrane raft, mitochondrial outer membrane, and region of cytosol. Furthermore, a total of 118 molecular functions were enriched; the top 10 entries were selected, containing steroid hormone receptor activity, monocarboxylic acid-binding, carboxylic acid-binding, fatty acid-binding, organic acid-binding, steroid binding, nuclear receptor activity, ligand-activated transcription factor activity, nuclear receptor transcription coactivator activity, and long-chain fatty acid-binding. These processes are of great significance to further understand the curative mechanism of Cordyceps on the treatment of DN. The results of the GO analysis are illustrated in Figure 6.

In terms of KEGG analysis, a total of 118 pathways were obtained. The top 20 significantly enriched pathways were screened out based on the threshold of $P<0.05$ (Figure 7). The results indicated that these genes were mainly associated with the inflammatory signaling pathway, apoptosis, oxidative stress, and insulin signaling pathway, including AGERAGE signaling pathway in diabetic complications, TNF signaling pathway, apoptosis, IL-17 signaling pathway, PI3KAkt signaling pathway, and insulin resistance. The results prove that Cordyceps may alleviate $\mathrm{DN}$ by regulating insulin resistance, apoptosis, and inflammatory reaction.

To more intuitively demonstrate which pathway each target is involved in, the target pathway data captured from KEGG analysis was uploaded into Cytoscape software for constructing a network graph of target and pathway (Figure 8).

\section{Discussion}

$\mathrm{DN}$, featured as high incidence, multifactorial pathogenesis, and absence of practical methods for diagnosis and treatment, is undoubtedly a medical challenge worldwide. The etiology of DN is multifactorial; with hyperglycemia, oxidative stress, and advanced glycation end products (AGE) as the leading factors, chronic inflammation and infiltrated immune cells in renal tissue are considered to be the common pathological consequences [20, 21]. Despite current improving therapies, there is still a considerable residual risk of DN onset and progression [22]. Some relevant studies highlight new perspectives of TCM for delaying DN progression and strengthen the therapeutic rationale for TCM on the treatment of DN [23,24]. Cordyceps contains several active ingredients which affect multiple targets and pathways in the progression of DN and has been used as an adjuvant on the treatment of DN in China for a long time $[7,25]$. In this study, we selected 7 active ingredients from Cordyceps based on the $\mathrm{OB}$ and DL, including linoleyl acetate, arachidonic acid, cholesteryl palmitate, CLR, beta-sitosterol, cerevisterol, and peroxyergosterol. Some compounds of Cordyceps were reported to have the effect on ameliorating endocrine and metabolic disorders during the development of DN [2629]. For instance, it was reported that beta-sitosterol protects the expression of insulin signaling molecules through activating insulin receptor and glucose transporter 4 in the adipose tissue with a high-fat diet, which can slow the development of DN [29]. Arachidonic acid is a strong inducer of insulin secretion and it can attenuate DN by inhibiting the TGF$\beta /$ Smad signaling pathway $[26,30]$. In addition, arachidonic acid also can facilitate the production of anti-inflammatory lipoxins which were reported to improve insulin sensitivity 
TABLE 2: Information of putative targets and topological attributes.

\begin{tabular}{|c|c|c|c|c|}
\hline No. & $\begin{array}{l}\text { UniProt } \\
\text { ID }\end{array}$ & Protein names & $\begin{array}{c}\text { Gene } \\
\text { names }\end{array}$ & Degree \\
\hline 1 & P01375 & Tumor necrosis factor & TNF & 51 \\
\hline 2 & P28482 & Mitogen-activated protein kinase 1 & MAPK1 & 45 \\
\hline 3 & P01133 & Proepidermal growth factor & EGF & 45 \\
\hline 4 & P00533 & Epidermal growth factor receptor & EGFR & 45 \\
\hline 5 & P42574 & Caspase-3 & CASP3 & 45 \\
\hline 6 & P04637 & Cellular tumor antigen p53 & TP53 & 44 \\
\hline 7 & P35354 & Prostaglandin G/H synthase 2 & PTGS2 & 41 \\
\hline 8 & P14780 & Matrix metalloproteinase- 9 & MMP9 & 40 \\
\hline 9 & P29474 & Nitric oxide synthase, endothelial & NOS3 & 40 \\
\hline 10 & P05412 & Transcription factor AP-1 & JUN & 40 \\
\hline 11 & P37231 & Peroxisome proliferator-activated receptor gamma & PPARG & 37 \\
\hline 12 & P60484 & $\begin{array}{c}\text { Phosphatidylinositol 3,4,5-trisphosphate 3-phosphatase and dual-specificity protein phosphatase } \\
\text { PTEN }\end{array}$ & PTEN & 37 \\
\hline 13 & P45983 & Mitogen-activated protein kinase 8 & MAPK8 & 36 \\
\hline 14 & Q04206 & Transcription factor p65 & RELA & 35 \\
\hline 15 & P35968 & Vascular endothelial growth factor receptor 2 & KDR & 33 \\
\hline 16 & Q16539 & Mitogen-activated protein kinase 14 & MAPK14 & 33 \\
\hline 17 & P08253 & $72 \mathrm{kDa}$ type IV collagenase & MMP2 & 32 \\
\hline 18 & P42345 & Serine/threonine-protein kinase mTOR & MTOR & 32 \\
\hline 19 & P03372 & Estrogen receptor & ESR1 & 31 \\
\hline 20 & P12821 & Angiotensin-converting enzyme & $\mathrm{ACE}$ & 28 \\
\hline 21 & P01137 & Transforming growth factor beta- 1 proprotein & TGFB1 & 27 \\
\hline 22 & P16284 & Platelet endothelial cell adhesion molecule & PECAM1 & 27 \\
\hline 23 & P42336 & Phosphatidylinositol 4,5-bisphosphate 3-kinase catalytic subunit alpha isoform & PIK3CA & 26 \\
\hline 24 & P08254 & Stromelysin-1 & MMP3 & 25 \\
\hline 25 & Q14790 & Caspase- 8 & CASP8 & 25 \\
\hline 26 & P09619 & Platelet-derived growth factor receptor beta & PDGFRB & 23 \\
\hline 27 & Q00987 & E3 ubiquitin-protein ligase $\mathrm{Mdm} 2$ & MDM2 & 23 \\
\hline 28 & P19438 & Tumor necrosis factor receptor superfamily member $1 \mathrm{~A}$ & TNFRSF1A & 23 \\
\hline 29 & P55211 & Caspase- 9 & CASP9 & 22 \\
\hline 30 & Q06124 & Tyrosine-protein phosphatase nonreceptor type 11 & PTPN11 & 22 \\
\hline 31 & P41235 & Hepatocyte nuclear factor 4-alpha & HNF4A & 20 \\
\hline 32 & P30556 & Type- 1 angiotensin II receptor & AGTR1 & 20 \\
\hline 33 & Q07869 & Peroxisome proliferator-activated receptor alpha & PPARA & 20 \\
\hline 34 & P11511 & Aromatase & CYP19A1 & 16 \\
\hline 35 & P35228 & Nitric oxide synthase, inducible & NOS2 & 16 \\
\hline 36 & P49327 & Fatty acid synthase & FASN & 16 \\
\hline 37 & O14920 & Inhibitor of nuclear factor kappa-B kinase subunit beta & IKBKB & 16 \\
\hline 38 & P25116 & Proteinase-activated receptor 1 & $\mathrm{~F} 2 \mathrm{R}$ & 15 \\
\hline 39 & P17252 & Protein kinase $\mathrm{C}$ alpha type & PRKCA & 15 \\
\hline 40 & P07148 & Fatty acid-binding protein, liver & FABP1 & 15 \\
\hline 41 & P15090 & Fatty acid-binding protein, adipocyte & FABP4 & 15 \\
\hline 42 & P16109 & P-selectin & SELP & 14 \\
\hline 43 & P11473 & Vitamin D3 receptor & VDR & 14 \\
\hline 44 & P29350 & Tyrosine-protein phosphatase nonreceptor type 6 & PTPN6 & 14 \\
\hline 45 & O95477 & Phospholipid-transporting ATPase ABCA1 & ABCA1 & 14 \\
\hline 46 & P25101 & Endothelin-1 receptor & EDNRA & 13 \\
\hline 47 & P55851 & Mitochondrial uncoupling protein 2 & UCP2 & 12 \\
\hline
\end{tabular}


TABle 2: Continued.

\begin{tabular}{|c|c|c|c|c|}
\hline No. & $\begin{array}{l}\text { UniProt } \\
\text { ID }\end{array}$ & Protein names & $\begin{array}{c}\text { Gene } \\
\text { names }\end{array}$ & Degree \\
\hline 48 & P48736 & Phosphatidylinositol 4,5-bisphosphate 3-kinase catalytic subunit gamma isoform & PIK3CG & 12 \\
\hline 49 & P05771 & Protein kinase $\mathrm{C}$ beta type & PRKCB & 12 \\
\hline 50 & Q13464 & Rho-associated protein kinase 1 & ROCK1 & 12 \\
\hline 51 & P20333 & Tumor necrosis factor receptor superfamily member $1 \mathrm{~B}$ & TNFRSF1B & 12 \\
\hline 52 & P15121 & Aldo-keto reductase family 1 member B1 & AKR1B1 & 11 \\
\hline 53 & P21554 & Cannabinoid receptor 1 & CNR1 & 11 \\
\hline 54 & P04629 & High affinity nerve growth factor receptor & NTRK1 & 11 \\
\hline 55 & P22894 & Neutrophil collagenase & MMP8 & 11 \\
\hline 56 & Q99814 & Endothelial PAS domain-containing protein 1 & EPAS1 & 10 \\
\hline 57 & P10415 & Apoptosis regulator Bcl-2 & BCL2 & 10 \\
\hline 58 & P32246 & $\mathrm{C}-\mathrm{C}$ chemokine receptor type 1 & CCR1 & 8 \\
\hline 59 & P11413 & Glucose-6-phosphate 1-dehydrogenase & G6PD & 8 \\
\hline 60 & P08123 & Collagen alpha-2 & COL1A2 & 8 \\
\hline 61 & P23219 & Prostaglandin G/H synthase 1 & PTGS1 & 8 \\
\hline 62 & Q13133 & Oxysterols receptor LXR-alpha & NR1H3 & 8 \\
\hline 63 & O75116 & Rho-associated protein kinase 2 & ROCK2 & 8 \\
\hline 64 & Q03181 & Peroxisome proliferator-activated receptor delta & PPARD & 7 \\
\hline 65 & P25105 & Platelet-activating factor receptor & PTAFR & 7 \\
\hline 66 & P27169 & Serum paraoxonase/arylesterase 1 & PON1 & 7 \\
\hline 67 & P17706 & Tyrosine-protein phosphatase nonreceptor type 2 & PTPN2 & 7 \\
\hline 68 & O75469 & Nuclear receptor subfamily 1 group I member 2 & NR1I2 & 6 \\
\hline 69 & P28223 & 5-Hydroxytryptamine receptor $2 \mathrm{~A}$ & HTR2A & 6 \\
\hline 70 & P12104 & Fatty acid-binding protein, intestinal & FABP2 & 6 \\
\hline 71 & P02753 & Retinol-binding protein 4 & RBP4 & 5 \\
\hline 72 & P39900 & Macrophage metalloelastase & MMP12 & 5 \\
\hline 73 & P08235 & Mineralocorticoid receptor & NR3C2 & 5 \\
\hline 74 & P11597 & Cholesteryl ester transfer protein & CETP & 5 \\
\hline 75 & P07477 & Trypsin-1 & PRSS1 & 4 \\
\hline 76 & Q07973 & 1,25-Dihydroxyvitamin D & CYP24A1 & 4 \\
\hline 77 & P30542 & Adenosine receptor A1 & ADORA1 & 4 \\
\hline 78 & P18054 & Polyunsaturated fatty acid lipoxygenase ALOX12 & ALOX12 & 4 \\
\hline 79 & O00763 & Acetyl-CoA carboxylase 2 & ACACB & 4 \\
\hline 80 & P04278 & Sex hormone-binding globulin & SHBG & 3 \\
\hline 81 & P80365 & Corticosteroid 11-beta-dehydrogenase isozyme 2 & HSD11B2 & 3 \\
\hline 82 & P13866 & Sodium/glucose cotransporter 1 & SLC5A1 & 2 \\
\hline 83 & P35610 & Sterol O-acyltransferase 1 & SOAT1 & 1 \\
\hline 84 & P05091 & Aldehyde dehydrogenase, mitochondrial & ALDH2 & 1 \\
\hline
\end{tabular}




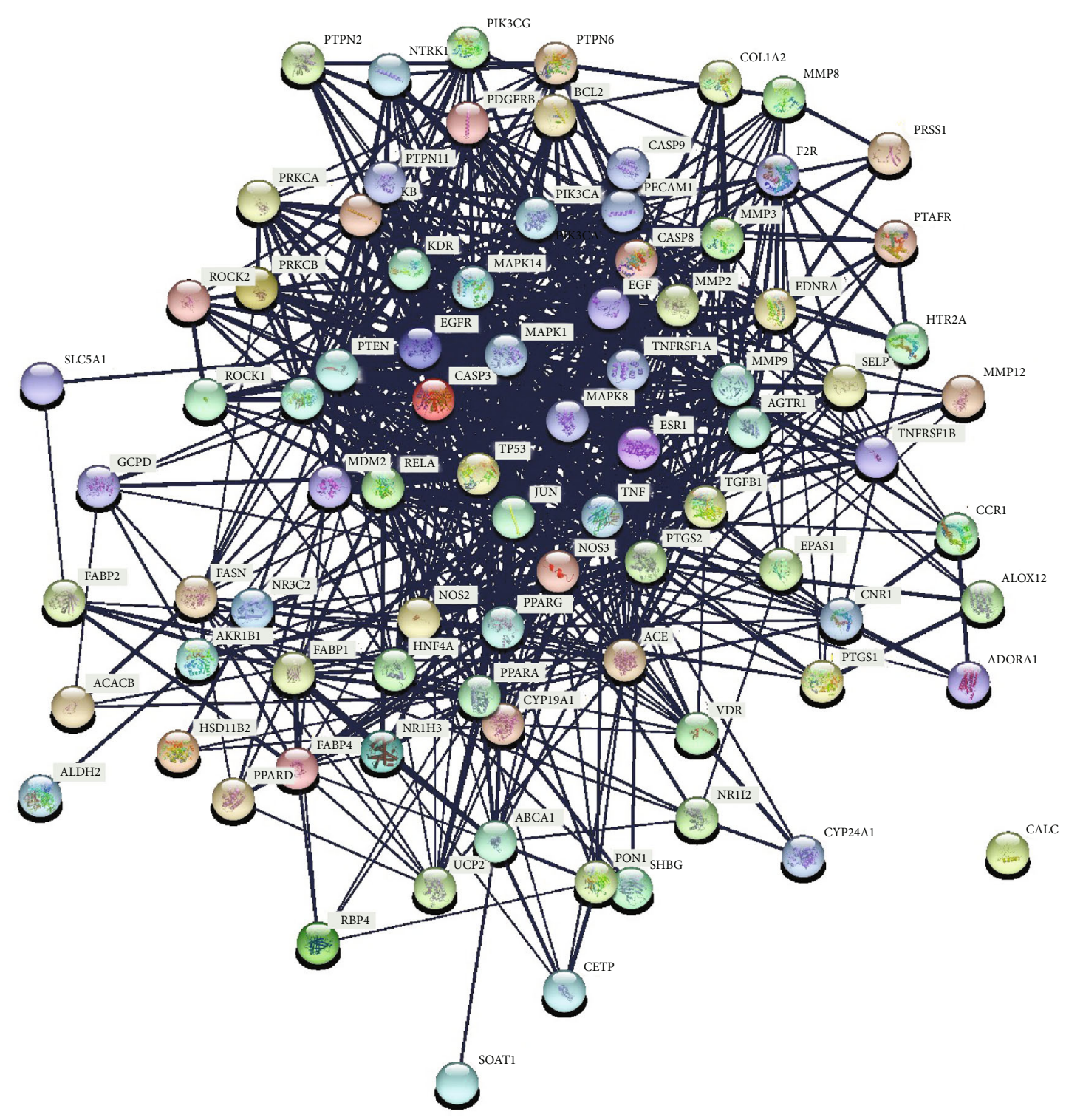

FIGURE 4: PPI network of overlapping targets between DN and Cordyceps. Each circular node represents a protein target, and the 3D structure in the circular nodes shows the protein spatial structure. The lines among different nodes represent the association among potential protein targets, while the width of lines was according to the action intensity.

and may prevent the development of diabetes [27]. Besides, several arachidonic acid metabolites, including PGE2, PGI2, and LXA4, PGE2 and PGI2 can alleviate insulin resistance and improve insulin sensitivity of pancreatic cells [28]; LXA 4 can inhibit the production of IL- 6 , TNF- $\alpha$, and ROS, thus alleviating inflammation, and has an antidiabetic effect $[31,32]$.

We found that many targets can be regulated by multiple compounds from the compound-target network, such as CYP19A1, NOS2, NR1H3, SHBG, and PTGS2. When it comes to core targets, TNF (degree=51), MAPK1 $($ degree $=51)$, EGFR $($ degree $=45)$, and CASP3 $($ degree $=45$ ) played an important role in the process of Cordyceps in DN treatment. TNF and MAPK1 are correlated with inflammation response and deterioration of renal function $[33,34]$. CASP3 is known to have an important role in the promotion of apoptotic cell death [35]. AG1478 can block EGFR signaling and inhibit oxidative stress and endoplasmic reticulum stress markers in diabetic mice [36]. In addition, inhibition of EGFR activation is associated with improved DN and insulin resistance in type 2 diabetes mouse models [37]. Therefore, the candidate targets are mainly enriched for oxidative stress, insulin resistance, apoptosis, and inflammation.

We also selected 85 common targets between the components of Cordyceps and DN for performing GO enrichment, consisting of biological processes, molecular function, and cellular components, which is aimed at predicting the mechanism of Cordyceps in treating $\mathrm{DN}$. We found that the candidate targets are involved in multiple biological processes, such as regulation of inflammatory response, response to lipopolysaccharide, response to 


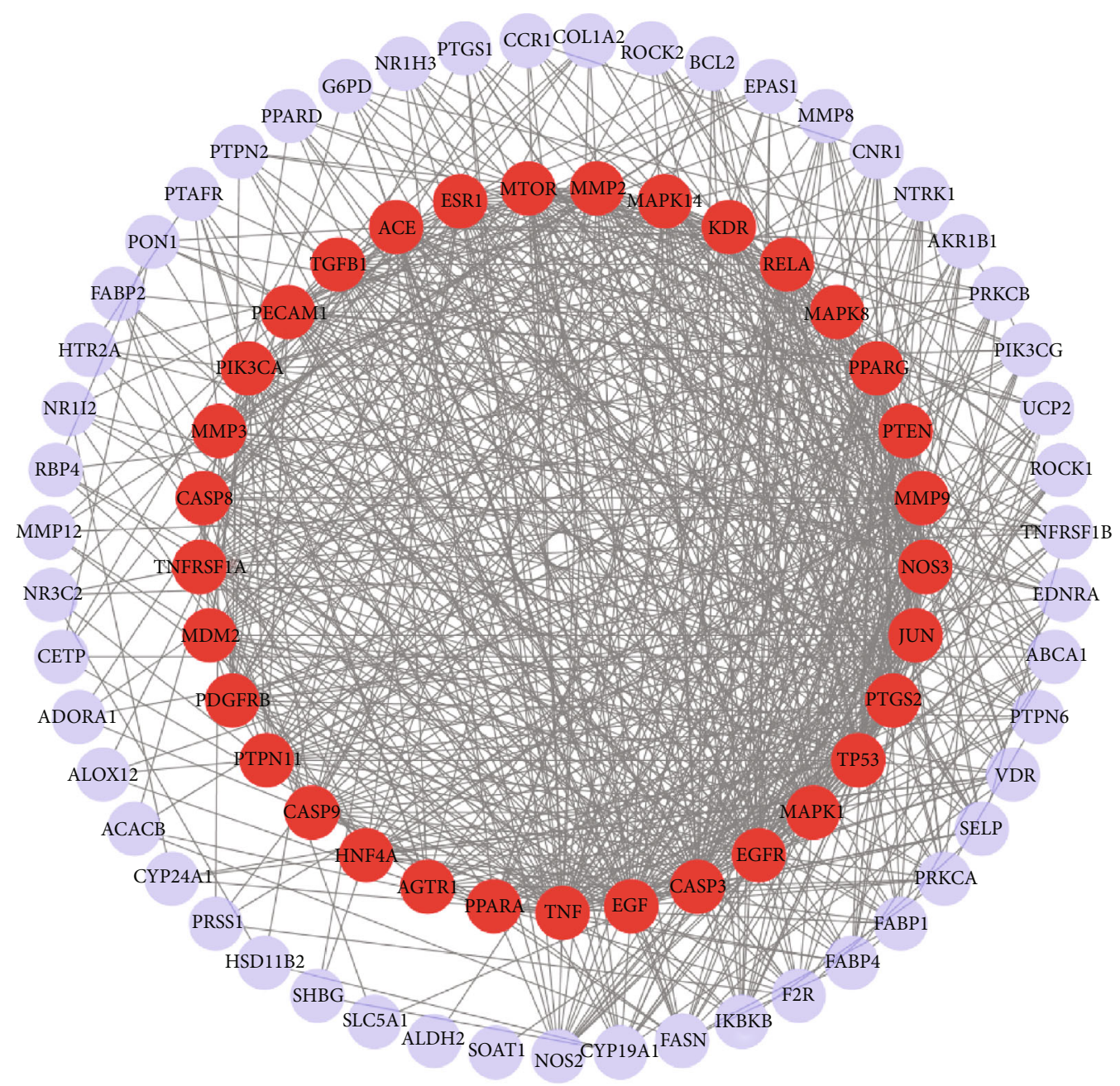

Figure 5: PPI network integration and analysis. The red nodes display the most potential targets in treating DN, which are greater than the average degree. Also, the nodes in purple show the potential targets under the average degree. Furthermore, the density of lines among inner nodes indicates the interaction relationship between different protein targets.

nutrient levels, response to oxygen levels, cellular response to chemical stress, regulation of lipid metabolic process, and lipid localization. The active targets such as TNF, PPARG, MAPK1, EGFR, and TGF- $\beta 1$ mainly participate in the biological processes of inflammatory response, oxidative stress, and lipid metabolic process [36, 38, 39]. Thus, the molecular processes of several targets are relatively consistent with the pathogenesis and mechanism of clinical DN. In addition, cellular components constitute membrane raft, membrane microdomain, membrane region, RNA polymerase II transcription regulator complex, and transcription regulator complex. It indirectly illustrates the complexity of the pathogenesis of DN and its damage to several cellular components, and Cordyceps may have a function in regulating these cellular components, eventually improving DN. Besides, molecular functions are mainly enriched in steroid hormone-related activity and steroid-binding, and many studies reported that steroid hormones were closely related to DN in patients with diabetes [40, 41]. It reveals that Cordyceps might target steroid hormone in DN treatment.

Using network pharmacological analysis and performing KEGG enrichment, we found that these Cordyceps components may relieve the symptoms of DN through the action of targets in various signaling pathways and multiple biological processes, including AGE-RAGE signaling pathway, TNF signaling pathway, apoptosis, IL-17 signaling pathway, PI3K-Akt signaling pathway, and insulin resistance. Activation of the receptor for AGEs or RAGE (receptor for advanced glycation end-products) is associated with the development of DN [42], which evokes oxidative stress and chronic inflammation in renal tissues, ending up in losses in kidney function by activating various intracellular signalings like PI3K/Akt/mTOR, NF- $\kappa$ B, MAPK/ERK, and TGF$\beta /$ Smad [43-45]. Additionally, it is believed that reactive oxygen species (ROS) can regulate PI3K/Akt/mTOR signaling and play an essential role in the development of DN, including epithelial-mesenchymal transition (EMT). During EMT, 


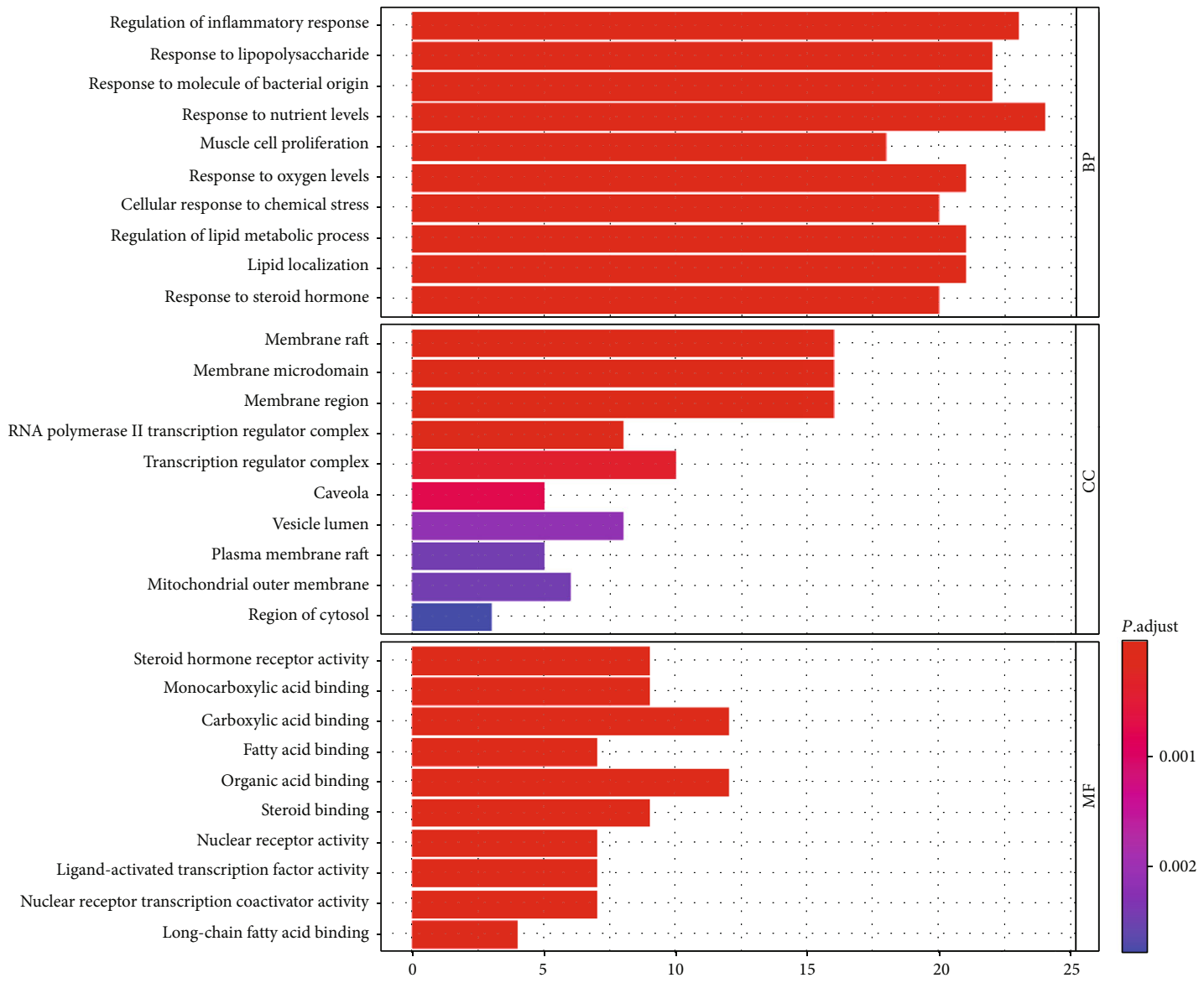

FIgURE 6: Enriched GO terms for BP, MF, and CC of putative targets of active components of Cordyceps. The color of the bar is displayed in a gradient from red to blue according to the ascending order of the $P$ value, while the length of the bar is arranged according to the ascending order of the number of gene counts.

epithelial cells lose their primary epithelial properties, such as epithelial- (E-) cadherin, while acquiring characteristics typical of mesenchymal cells such as $\alpha$-SMA, ending up with renal interstitial fibrosis [46]. Moreover, PI3K/Akt/mTOR signaling can promote high glucose-induced podocyte apoptosis, which contributes to the pathogenesis of DN [47].

TNF signaling is characterized as a well-known inflammatory cytokine associated with renal injury [48]. Once stimulated by TNF- $\alpha$, NF- $\kappa$ B moves from the cytoplasm to the nucleus and activates the transcription of VCAM-1, ICAM1, IL-6, and IL-8, which will result in endothelial inflammatory and DN pathological process acceleration [49]. Furthermore, NF- $\kappa \mathrm{B}$ can be activated by several cytokines, which in turn induces the production of TNF- $\alpha$, resulting in diabetic renal damage [50].

Insulin resistance is a critical process and one of the main symptoms in the initiation and progression of $\mathrm{DN}$, which is closely related to microalbuminuria [51, 52]. High levels of insulin can cause insulin receptor degradation and drive early podocyte insulin resistance, and both the insulin receptor and nephrin are needed for full insulin sensitivity of podocytes. Thus, it explains why individuals with nephropathy caused by type 2 diabetes are commonly hyperinsulinaemic in the early stage of their disease [53]. Moreover, there are lots of mediators of insulin resistance that participate in driving renal function decline, including TGF- $\beta 1$, blood pressure, inflammation, TNF- $\alpha$, IL-6, and oxidative stress [5456].

Therefore, these results of network pharmacological analyses not only verify that our screened targets are consistent with previous literature reports but also indicate that Cordyceps play a significant therapeutic role in DN by regulating several signaling pathways, including inflammatory response, insulin resistance, oxidative stress, apoptosis, and other pathways with unclear mechanisms. It will provide a novel methodology for further study of the therapeutic mechanism of Cordyceps in alleviating DN.

\section{Conclusion}

In summary, our network pharmacological analyses have shown that Cordyceps play an indispensable supplementary role in the treatment of $\mathrm{DN}$, which is consistent with previous studies. Moreover, the biological functions of active chemical molecules and their corresponding targets of Cordyceps analyzed by network pharmacological methods provide a unique and innovative path for the 


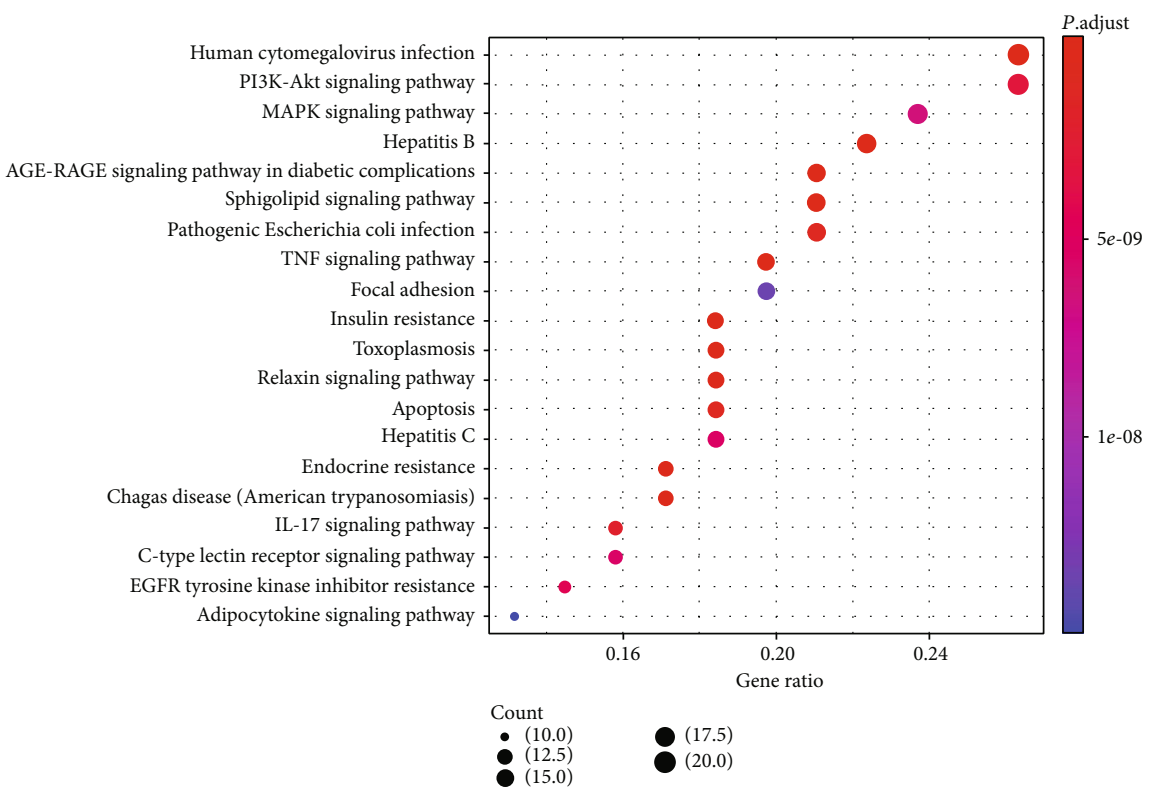

Figure 7: KEGG pathway analysis of potential targets of Cordyceps. The top 20 pathways are selected following the criteria of $P<0.05$. The longitudinal axis represents the name of different pathways, and the transverse axis shows the number of enriched genes. Besides, the dot size represents the proportion of the number of enriched genes to the total number of genes, which is according to the descending proportion value. And the color of the dot is displayed in a gradient from red to blue according to the ascending order of the $P$ value.

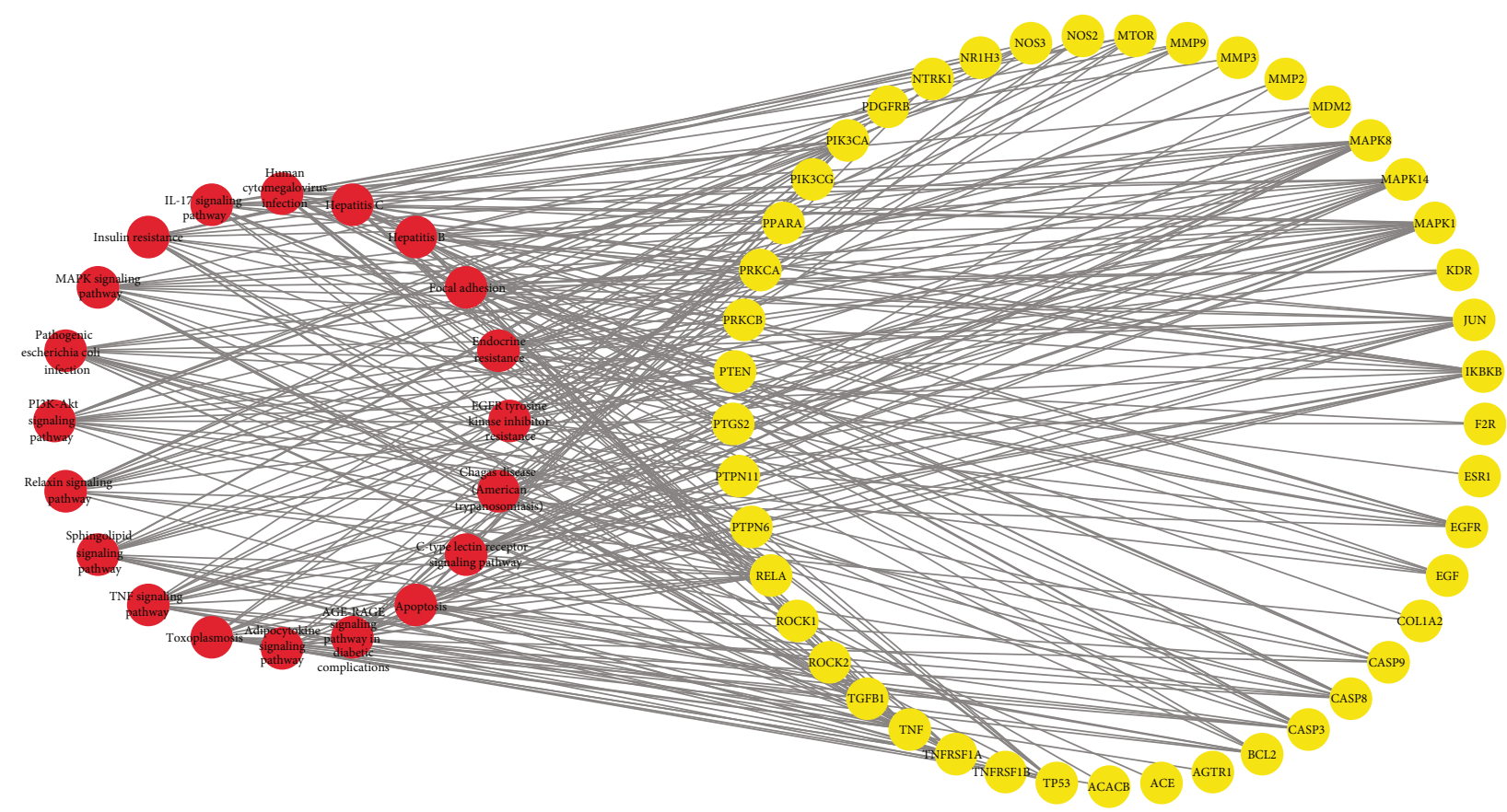

FIGURE 8: Pathway-target interaction network. Red circles indicate pathways which have interactions with potential targets, while the yellow circles represent putative targets.

study of TCM and further reveal the molecular biological mechanism of Cordyceps in treating DN. However, in vivo and in vitro experiments should be undertaken to validate the relationship between key targets and pathways of Cordyceps for the treatment of DN. Despite the limitations of this study, the results of this study provide new evidence and theoretical basis which will be used in subsequent theoretical and clinical research studies of Cordyceps.

\section{Data Availability}

The data used to support the findings of this study are included within the article. 


\section{Conflicts of Interest}

The authors declare that they have no competing interests.

\section{Authors' Contributions}

Yan Li and Lei Wang contributed equally to this work. All authors have contributed to this study and approve its submission.

\section{Acknowledgments}

This work was supported by the Science and Technology Department of Sichuan Province (grant number: 20YYJC4065).

\section{References}

[1] M. K. Sagoo and L. Gnudi, "Diabetic nephropathy: an overview," Methods in Molecular Biology, vol. 2067, pp. 3-7, 2020.

[2] N. Raval, A. Kumawat, D. Kalyane, K. Kalia, and R. K. Tekade, "Understanding molecular upsets in diabetic nephropathy to identify novel targets and treatment opportunities," Drug Discovery Today, vol. 25, no. 5, pp. 862-878, 2020.

[3] H. Fu, S. Liu, S. I. Bastacky, X. Wang, X. J. Tian, and D. Zhou, "Diabetic kidney diseases revisited: a new perspective for a new era," Mol Metab, vol. 30, pp. 250-263, 2019.

[4] G.-d. Sun, C.-y. Li, W.-p. Cui et al., "Review of herbal traditional Chinese medicine for the treatment of diabetic nephropathy," Journal Diabetes Research, vol. 2016, article 5749857, pp. 1-18, 2016.

[5] O. J. Olatunji, J. Tang, A. Tola, F. Auberon, O. Oluwaniyi, and Z. Ouyang, "The genus Cordyceps: an extensive review of its traditional uses, phytochemistry and pharmacology," Fitoterapia, vol. 129, pp. 293-316, 2018.

[6] J. Yang, H. Dong, Y. Wang et al., "Cordyceps cicadae polysaccharides ameliorated renal interstitial fibrosis in diabetic nephropathy rats by repressing inflammation and modulating gut microbiota dysbiosis," International Journal of Biological Macromolecules, vol. 163, pp. 442-456, 2020.

[7] Z. Dong, Y. Sun, G. Wei, S. Li, and Z. Zhao, "A nucleoside/nucleobase-rich extract from Cordyceps sinensis inhibits the epithelial-mesenchymal transition and protects against renal fibrosis in diabetic nephropathy," Molecules, vol. 24, no. 22, p. $4119,2019$.

[8] D. D. Chen, R. Xu, J. Y. Zhou et al., "Cordyceps militaris polysaccharides exerted protective effects on diabetic nephropathy in mice via regulation of autophagy," Food \& Function, vol. 10, no. 8, pp. 5102-5114, 2019.

[9] X. Wang, A. Qin, F. Xiao et al., "N6-(2-Hydroxyethyl)-adenosine fromCordyceps cicadaeprotects against diabetic kidney disease via alleviation of oxidative stress and inflammation," Journal of Food Biochemistry, vol. 43, no. 2, article e12727, 2019.

[10] J. Ru, P. Li, J. Wang et al., "TCMSP: a database of systems pharmacology for drug discovery from herbal medicines," Journal of Cheminformatics, vol. 6, no. 1, p. 13, 2014.

[11] S. Kim, "Getting the most out of PubChem for virtual screening," Expert Opin Drug Discov, vol. 11, no. 9, pp. 843-855, 2016.
[12] D. Gfeller, A. Grosdidier, M. Wirth, A. Daina, O. Michielin, and V. Zoete, "SwissTargetPrediction: a web server for target prediction of bioactive small molecules," Nucleic Acids Res, vol. 42, pp. W32-W38, 2014.

[13] N. T. Doncheva, J. H. Morris, J. Gorodkin, and L. J. Jensen, "Cytoscape StringApp: network analysis and visualization of proteomics data," Journal of Proteome Research, vol. 18, no. 2, pp. 623-632, 2018.

[14] G. Stelzer, N. Rosen, I. Plaschkes et al., "The GeneCards suite: from gene data mining to disease genome sequence analyses," Current Protocols in Bioinformatics, vol. 54, pp. 1.30.1-1.30.33, 2016.

[15] J. Piñero, À. Bravo, N. Queralt-Rosinach et al., "DisGeNET: a comprehensive platform integrating information on human disease-associated genes and variants," Nucleic Acids Research, vol. 45, no. D1, pp. D833-d839, 2017.

[16] M. Pathan, S. Keerthikumar, C. S. Ang et al., "FunRich: an open access standalone functional enrichment and interaction network analysis tool," Proteomics, vol. 15, no. 15, pp. 25972601, 2015.

[17] D. Szklarczyk, A. L. Gable, D. Lyon et al., "STRING v11: protein-protein association networks with increased coverage, supporting functional discovery in genome-wide experimental datasets," Nucleic Acids Research, vol. 47, no. D1, pp. D607d613, 2019.

[18] The Gene Ontology Resource, "20 years and still GOing strong," Nucleic Acids Research, vol. 47, no. D1, pp. D330d338, 2019.

[19] M. Kanehisa, M. Furumichi, M. Tanabe, Y. Sato, and K. Morishima, "KEGG: new perspectives on genomes, pathways, diseases and drugs," Nucleic Acids Research, vol. 45, no. D1, pp. D353-d361, 2017.

[20] F. B. Hickey and F. Martin, "Role of the immune system in diabetic kidney disease," Current Diabetes Reports, vol. 18, no. 4, p. 20, 2018.

[21] G. H. Tesch, "Diabetic nephropathy - is this an immune disorder?," Clinical Science (London, England), vol. 131, no. 16, pp. 2183-2199, 2017.

[22] R. Z. Alicic, M. T. Rooney, and K. R. Tuttle, "Diabetic kidney disease: challenges, progress, and possibilities," Clinical Journal of the American Society of Nephrology, vol. 12, no. 12, pp. 2032-2045, 2017.

[23] H. Y. Chen, H. C. Pan, Y. C. Chen et al., "Traditional Chinese medicine use is associated with lower end-stage renal disease and mortality rates among patients with diabetic nephropathy: a population-based cohort study," BMC Complementary and Alternative Medicine, vol. 19, no. 1, p. 81, 2019.

[24] D. Wen, R. Z. Tan, C. Y. Zhao et al., "Astragalus mongholicus Bunge and Panax notoginseng (Burkill) F.H. Chen formula for renal injury in diabetic nephropathy-in vivo and in vitro evidence for autophagy regulation," Frontiers in Pharmacology, vol. 11, p. 732, 2020.

[25] K. Zhao, Q. Gao, C. Zong, L. Ge, and J. Liu, "Cordyceps sinensis prevents contrast-induced nephropathy in diabetic rats: its underlying mechanism," International Journal of Clinical and Experimental Pathology, vol. 11, no. 12, pp. 5571-5580, 2018.

[26] T. Sonnweber, A. Pizzini, M. Nairz, G. Weiss, and I. Tancevski, "Arachidonic acid metabolites in cardiovascular and metabolic diseases," International Journal of Molecular Sciences, vol. 19, no. 11 , p. 3285, 2018. 
[27] N. K. V. Gundala, V. G. M. Naidu, and U. N. Das, "Amelioration of streptozotocin-induced type 2 diabetes mellitus in Wistar rats by arachidonic acid," Biochemical and Biophysical Research Communications, vol. 496, no. 1, pp. 105-113, 2018.

[28] P. Luo and M. H. Wang, "Eicosanoids, $\beta$-cell function, and diabetes," Prostaglandins \& Other Lipid Mediators, vol. 95, no. 1-4, pp. 1-10, 2011.

[29] R. Ponnulakshmi, B. Shyamaladevi, P. Vijayalakshmi, and J. Selvaraj, "In silico and in vivo analysis to identify the antidiabetic activity of beta sitosterol in adipose tissue of high fat diet and sucrose induced type-2 diabetic experimental rats," Toxicology Mechanisms and Methods, vol. 29, no. 4, pp. 276-290, 2019.

[30] G. Chen, P. Wang, G. Zhao et al., "Cytochrome P450 epoxygenase CYP2J2 attenuates nephropathy in streptozotocininduced diabetic mice," Prostaglandins \& Other Lipid Mediators, vol. 96, no. 1-4, pp. 63-71, 2011.

[31] U. N. Das, "Arachidonic acid and lipoxin A4 as possible endogenous anti-diabetic molecules," Prostaglandins, Leukotrienes, and Essential Fatty Acids, vol. 88, no. 3, pp. 201-210, 2013.

[32] E. Börgeson, A. M. F. Johnson, Y. S. Lee et al., "Lipoxin A4 attenuates obesity-induced adipose inflammation and associated liver and kidney disease," Cell Metabolism, vol. 22, no. 1, pp. 125-137, 2015.

[33] J. F. Navarro and C. Mora-Fernández, “The role of TNF-alpha in diabetic nephropathy: pathogenic and therapeutic implications," Cytokine \& Growth Factor Reviews, vol. 17, no. 6, pp. 441-450, 2006.

[34] X. Q. Geng, A. Ma, J. Z. He et al., "Ganoderic acid hinders renal fibrosis via suppressing the TGF- $\beta /$ Smad and MAPK signaling pathways," Acta Pharmacologica Sinica, vol. 41, no. 5, pp. 670-677, 2020.

[35] B. Yang, S. Jain, S. Y. Ashra, P. N. Furness, and M. L. Nicholson, "Apoptosis and caspase-3 in long-term renal ischemia/reperfusion injury in rats and divergent effects of immunosuppressants," Transplantation, vol. 81, no. 10, pp. 1442-1450, 2006.

[36] Z. Xu, Y. Zhao, P. Zhong et al., "EGFR inhibition attenuates diabetic nephropathy through decreasing ROS and endoplasmic reticulum stress," Oncotarget, vol. 8, no. 20, pp. 3265532667, 2017.

[37] Z. Li, Y. Li, J. M. Overstreet et al., "Inhibition of epidermal growth factor receptor activation is associated with improved diabetic nephropathy and insulin resistance in type 2 diabetes," Diabetes, vol. 67, no. 9, pp. 1847-1857, 2018.

[38] L. S. Araújo, B. G. S. Torquato, C. A. da Silva et al., "Renal expression of cytokines and chemokines in diabetic nephropathy," BMC Nephrology, vol. 21, no. 1, p. 308, 2020.

[39] R. Yu, H. Bo, and S. Huang, "Association between the PPARG gene polymorphism and the risk of diabetic nephropathy: a meta-analysis," Genetic Testing and Molecular Biomarkers, vol. 16, no. 5, pp. 429-434, 2012.

[40] C. Maric, C. Forsblom, L. Thorn, J. Wadén, P. H. Groop, and FinnDiane Study Group, "Association between testosterone, estradiol and sex hormone binding globulin levels in men with type 1 diabetes with nephropathy," Steroids, vol. 75, no. 11, pp. 772-778, 2010.

[41] M. Grossmann, M. C. Thomas, S. Panagiotopoulos et al., "Low testosterone levels are common and associated with insulin resistance in men with diabetes," The Journal of Clinical Endocrinology and Metabolism, vol. 93, no. 5, pp. 1834-1840, 2008.
[42] J. M. Wadén, E. H. Dahlström, N. Elonen et al., "Soluble receptor for AGE in diabetic nephropathy and its progression in Finnish individuals with type 1 diabetes," Diabetologia, vol. 62, no. 7, pp. 1268-1274, 2019.

[43] J.-N. Hong, W.-W. Li, L.-L. Wang et al., "Jiangtang decoction ameliorate diabetic nephropathy through the regulation of PI3K/Akt-mediated NF- $\kappa$ B pathways in KK-Ay mice," Chinese Medicine, vol. 12, no. 1, p. 13, 2017.

[44] W. H. Yiu, D. W. L. Wong, H. J. Wu et al., "Kallistatin protects against diabetic nephropathy in $\mathrm{db} / \mathrm{db}$ mice by suppressing AGE-RAGE-induced oxidative stress," Kidney International, vol. 89, no. 2, pp. 386-398, 2016.

[45] D. Xu and J. M. Kyriakis, "Phosphatidylinositol 3'-kinasedependent activation of renal mesangial cell Ki-Ras and ERK by advanced glycation end products," The Journal of Biological Chemistry, vol. 278, no. 41, pp. 39349-39355, 2003.

[46] Q. Lu, W. W. Wang, M. Z. Zhang et al., "ROS induces epithelial-mesenchymal transition via the TGF$\beta 1 / \mathrm{PI}$ K/Akt/mTOR pathway in diabetic nephropathy," Experimental and Therapeutic Medicine, vol. 17, no. 1, pp. 835-846, 2019.

[47] X. M. Wang, M. Yao, S. X. Liu, J. Hao, Q. J. Liu, and F. Gao, "Interplay between the Notch and PI3K/Akt pathways in high glucose-induced podocyte apoptosis," American Journal of Physiology. Renal Physiology, vol. 306, no. 2, pp. F205-F213, 2014.

[48] J. F. Navarro, C. Mora, M. Muros, and J. Garcia, "Urinary tumour necrosis factor- excretion independently correlates with clinical markers of glomerular and tubulointerstitial injury in type 2 diabetic patients," Nephrology, Dialysis, Transplantation, vol. 21, no. 12, pp. 3428-3434, 2006.

[49] J. Liang, S. Yuan, X. Wang et al., "Attenuation of pristimerin on TNF- $\alpha$-induced endothelial inflammation," International Immunopharmacology, vol. 82, p. 106326, 2020.

[50] J. Caamaño and C. A. Hunter, "NF-kappaB family of transcription factors: central regulators of innate and adaptive immune functions," Clinical Microbiology Reviews, vol. 15, no. 3, pp. 414-429, 2002.

[51] A. I. Parvanova, R. Trevisan, I. P. Iliev et al., "Insulin resistance and microalbuminuria: a cross-sectional, case-control study of 158 patients with type 2 diabetes and different degrees of urinary albumin excretion," Diabetes, vol. 55, no. 5, pp. 14561462, 2006.

[52] P. A. Sarafidis and L. M. Ruilope, "Insulin resistance, microalbuminuria, and chronic kidney disease," Current Hypertension Reports, vol. 10, no. 4, pp. 249-251, 2008.

[53] A. C. Lay, J. A. Hurcombe, V. M. S. Betin et al., "Prolonged exposure of mouse and human podocytes to insulin induces insulin resistance through lysosomal and proteasomal degradation of the insulin receptor," Diabetologia, vol. 60, no. 11, pp. 2299-2311, 2017.

[54] J. Karalliedde and L. Gnudi, "Diabetes mellitus, a complex and heterogeneous disease, and the role of insulin resistance as a determinant of diabetic kidney disease," Nephrology, Dialysis, Transplantation, vol. 31, no. 2, pp. 206-213, 2016.

[55] S. E. Shoelson, J. Lee, and A. B. Goldfine, "Inflammation and insulin resistance," The Journal of Clinical Investigation, vol. 116, no. 7, pp. 1793-1801, 2006.

[56] L. Gnudi, "Cellular and molecular mechanisms of diabetic glomerulopathy," Nephrology, Dialysis, Transplantation, vol. 27, no. 7, pp. 2642-2649, 2012. 